\title{
Possibility of early diagnosis in a fetus affected by Prader-Willi syndrome with maternal hetero-UPD15: A lesson to be learned
}

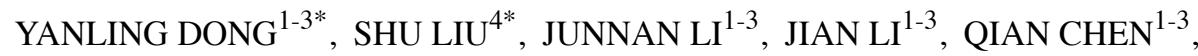 \\ JIANYUN LUO ${ }^{1-3}$, CHUNLEI LI ${ }^{1-3}$, HUIFAN LI ${ }^{1-3}$, HONGBO QI ${ }^{1-3}$ and RONG LI ${ }^{1-3}$ \\ ${ }^{1}$ Department of Obstetrics, The First Affiliated Hospital of Chongqing Medical University; ${ }^{2}$ State Key Laboratory of \\ Maternal and Fetal Medicine of Chongqing Municipality; ${ }^{3}$ International Collaborative Laboratory of Reproduction \\ and Development of Chinese Ministry of Education, Chongqing Medical University, Chongqing 400016; \\ ${ }^{4}$ Children Inherited Metabolism and Endocrine Department, Guangdong Women and \\ Children Hospital, Guangzhou, Guangdong 511400, P.R. China
}

Received October 15, 2018; Accepted April 5, 2019

DOI: $10.3892 / \mathrm{mmr} .2019 .10246$

\begin{abstract}
Prader-Willi syndrome (PWS), a complicated neurodevelopmental disorder arising from errors in genomic imprinting, is characterized by evident hypotonia along with feeding difficulties and the absence of crying in early infancy. Hyperphagia and obesity are not uncommon in patients with PWS, usually accompanied by intellectual disability, cognitive impairment, short stature, small hands and feet, as well as hypogonadism and typical facial features. Due to the severe complications associated with PWS, a thorough understanding of its features and an early diagnosis, preferably in the fetal period, are important for clinical management. According to previous studies, prenatal diagnosis has been confirmed in only a few cases of PWS, using ultrasound, or as an accidental finding by cytogenetic molecular techniques, as no precise fetal phenotype has been defined. In this present study, an infant with PWS arising from maternal heterodisomy of chromosome 15 is described. This is a typical case of missed diagnosis by fetal ultrasound examination, chromosome karyotype analysis and chromosome microarray (CMA) conducted during the pregnancy. To delineate the complex prenatal characteristics of a fetus with PWS, prenatally-diagnosed cases of PWS described in the literature were reviewed. This present study indicated that although prenatal signs are not sufficient for a diagnosis to be confirmed, a
\end{abstract}

Correspondence to: Professor Hongbo Qi or Professor Rong Li, Department of Obstetrics, The First Affiliated Hospital of Chongqing Medical University, 1 Youyi Road, Yuzhong, Chongqing 400016, P.R. China

E-mail: qihongbo728@163.com

E-mail: cqums.lirong@163.com

*Contributed equally

Key words: Prader-Willi syndrome, early diagnosis, prenatal diagnosis, developmental delay, chromosomal microarray analysis comprehensive consideration of these signs is important in leading to a diagnosis of suspected PWS, and thus prompts further prenatal investigations using molecular genetic tools. Furthermore, this present study also suggested that CMA can lead to a missed diagnosis of PWS/Angelman syndrome and other imprinting disorders despite its high value in the detection of copy-number variants in individuals with developmental delay. If clinical signs strongly suggest PWS, other prenatal molecular genetic investigations, including methylation tests and short tandem repeat-based linkage analysis for uniparental disomy, are recommended as an additional tool to aid diagnosis.

\section{Introduction}

Prader-Willi syndrome (PWS; OMIM 176270) was first reported by Prader et al (1) in 1956. As a complicated neurodevelopmental genetic disorder, PWS classically presents with severe hypotonia and feeding difficulties in the neonatal period, sometimes with concurrent anorexia and a sucking deficit (2). Challenged by the difficulties in early diagnosis, care and treatment, the majority of patients gain excessive weight at three or four years of age, and begin to exhibit signs of developmental delay and hypogonadism, eventually leading to severe early obesity, intellectual disability, cognitive impairment, genital hypoplasia and infertility $(3,4)$. PWS is characterized by a sporadic occurrence, which does not discriminate among gender or race, with an estimated prevalence of 1 in 15,000-30,000 live births (5). An interstitial deletion of paternal origin at 15q11.2-q13 may result in the absence of paternal expression of imprinted genes located in this chromosomal region. An imprinting defect (ID) or maternal uniparental disomy (UPD) of chromosome 15 may also be an explanation for the pathogenic mechanism of this disease (6). Due to the severe complications and high mortality of PWS, a multidisciplinary strategy is required to aid early diagnosis and optimize clinical management in order to improve quality of life and prognosis. Pediatricians worldwide are focusing effort on prenatal diagnosis. However, some clinical aspects of PWS in utero, including abnormal 
extremity positions, fetal hypomobility and polyhydramnios, can only guide PWS diagnosis owing to the lack of high specificity and sensitivity of these features. To date, there have been few reports examining the prenatal diagnosis of PWS using ultrasound or as an accidental finding by cytogenetic molecular techniques (7-9). In this present report, the case of an infant with PWS resulting from complete maternal heterodisomy (hetero-UPD) of chromosome 15 is presented. This case was not identified by fetal ultrasound examination, chromosome karyotype analysis or chromosome microarray (CMA) conducted during the pregnancy, but was identified retrospectively by postnatal diagnosis of the syndrome according to fetal ultrasound findings, postnatal clinical features and molecular genetic investigations.

\section{Patients and methods}

Clinical history. The 1-day-old male proband was the second child of healthy, non-consanguineous parents of 41 years of age. The family history was unremarkable. The parents had a healthy daughter of 10 years of age. During the pregnancy, polyhydramnios and a lack of fetal movements were noted. At 23 weeks and 6 days of gestation, ultrasound screening showed normal fetal growth with no structural abnormalities, but revealed polyhydramnios (amniotic fluid index, $25.2 \mathrm{~cm}$ ) accompanied by reduced fetal movement. At 30 weeks and 6 days of gestation, polyhydramnios (amniotic fluid index, $29.0 \mathrm{~cm}$ ) and decreased fetal activity persisted. Fetal biometric measurements confirmed that the fetal weight, estimated according to the abdominal circumference, head circumference and femoral length, was $\sim 1,180 \mathrm{~g}$, which was below the 10th percentile for this gestational age. Considering the advanced maternal age and polyhydramnios, interventional prenatal diagnosis was recommended by obstetricians. With a thorough understanding of sampling procedures, the potential risks and limitations of the test, the couple agreed to amniocentesis at 24 weeks plus 2 days of gestation for routine chromosome examination and CMA. Giemsa banded chromosome analysis of amniotic fluid cells cultured in situ and CMA revealed an apparently normal karyotype of 46, XY (data not shown), and the pregnancy was continued. At 38 weeks plus 1 day of gestation, the mother was hospitalized with fetal distress. An elective caesarean section was performed on the day of admission (October 2017) due to breech presentation. The caesarean was performed in The Obstetrical Department of The First Affiliated Hospital of Chongqing Medical University. The neonatal evaluation recorded a birth weight of $2,680 \mathrm{~g}$ (5th percentile), a length of $47 \mathrm{~cm}$ (2.9th percentile), and a head circumference of $33.5 \mathrm{~cm}$ (20.7th percentile), with an appearance, pulse, grimace, activity and respiration score of 9,10 and 10 at 1,5 and $10 \mathrm{~min}(10,11)$, respectively. The boy was admitted to the neonatal intensive care unit directly after birth due to a severely poor suck and hypotonia. Notable physical features included a narrow forehead, up-slanted palpebral fissure, bilateral epicanthus, micrognathia, wide spaced nipples, long slender fingers, hypopigmentation, a weak cry and hypoplastic external genitalia (Fig. 1). A neurological exam showed significant hypotonia. Gastric tube feeding was required due to the poor sucking. Clinically, the physical features were indicative of PWS. In view of the negative results of prenatal cytogenetic and molecular analysis, rare types of PWS, including maternal heterodisomy and ID were considered. On day 5 after birth, specific genetic examinations were conducted. At 1 month of age, with a body weight of $3,160 \mathrm{~g}$ ( 0.9 th percentile), a length of $48.5 \mathrm{~cm}$ (0.1th percentile) and a head circumference of $35.2 \mathrm{~cm}(8 \mathrm{th}$ percentile), the boy was evaluated again by geneticists. Special feeding techniques were still required due to the persistent difficulty in mouth feeding and poor weight gain. On physical examination, hypotonia remained and symptoms of upper respiratory infections, including cough, fever and nasal discharge, were noticed. The boy succumbed to from recurrent respiratory infections, hypoventilation and food choking at 4 months of age.

Methylation status analysis. In order to confirm the diagnosis of PWS, a DNA methylation test was conducted in which peripheral blood lymphocytes were collected from $2 \mathrm{ml}$ blood. To isolate genomic DNA, the QIAamp DNA blood mini kit (cat. no. 51104; Qiagen $\mathrm{GmbH}$ ) was used according to the manufacturer's protocol. The DNA samples were treated with the CpGenome ${ }^{\mathrm{TM}}$ Turbo Bisulfite Modification kit (cat. no. S7820; Merck KGaA) following the manufacturer's protocol. The DNA modified by bisulfite was amplified with primers specific to the differentially methylated sites within exon 1 and the promoter regions of the gene encoding small nuclear ribonucleoprotein polypeptide N: Methylated-specific forward, 5'-TAAATA AGTACGTTTGCGCGGTC-3' and reverse, 5'-AACCTTACCCGCTCCATCGCG-3' amplified a 174-bp DNA region, and non-methylated-specific forward, 5'-GTAGGTTGGTGTGTATGTTTAGGT-3' and reverse, 5'-ACATCAAACATCTCCAACAACCA-3' were used to amplify a 100-bp DNA region. Methylation-specific (MS) PCR was carried out as described in previous studies $(12,13)$. Amplification products were compared with appropriate positive (patients with PWS) and negative (healthy patients) controls, using agarose gel electrophoresis. The results showed two PCR products of 174 and $100 \mathrm{bp}$ in unaffected individuals. By contrast, only the 174 bp product from the maternal allele was observed, with the absence of the 100 bp product in this patient with PWS.

Chromosomal microarray analysis. Following salt-induced precipitation, DNA from peripheral blood samples was genotyped using CytoScan HD array (Affymetrix; Thermo Fisher Scientific, Inc.). In this experiment, genotype calling, quality control and identification of copy-number variation (CNV) were performed using Affymetrix Chromosome Analysis Suite software (version 4.0; Affymetrix; Thermo Fisher Scientific, Inc.), with various databases employed for evaluation of the array data and analysis of genotype-phenotype correlations, including OMIM (http://www.ncbi.nlm.nih. gov/omim), DECIPHER (http://decipher.sanger.ac.uk/), DGV (http://projects.tcag.ca/variation) and ISCA (http://dbsearch. clinicalgenome.org/search/).

UPD classification with the UPDtool. All CNVs were excluded before analysis to prevent their potential interference with UPD detection. A UPD converter tool (UPDtool; 
A

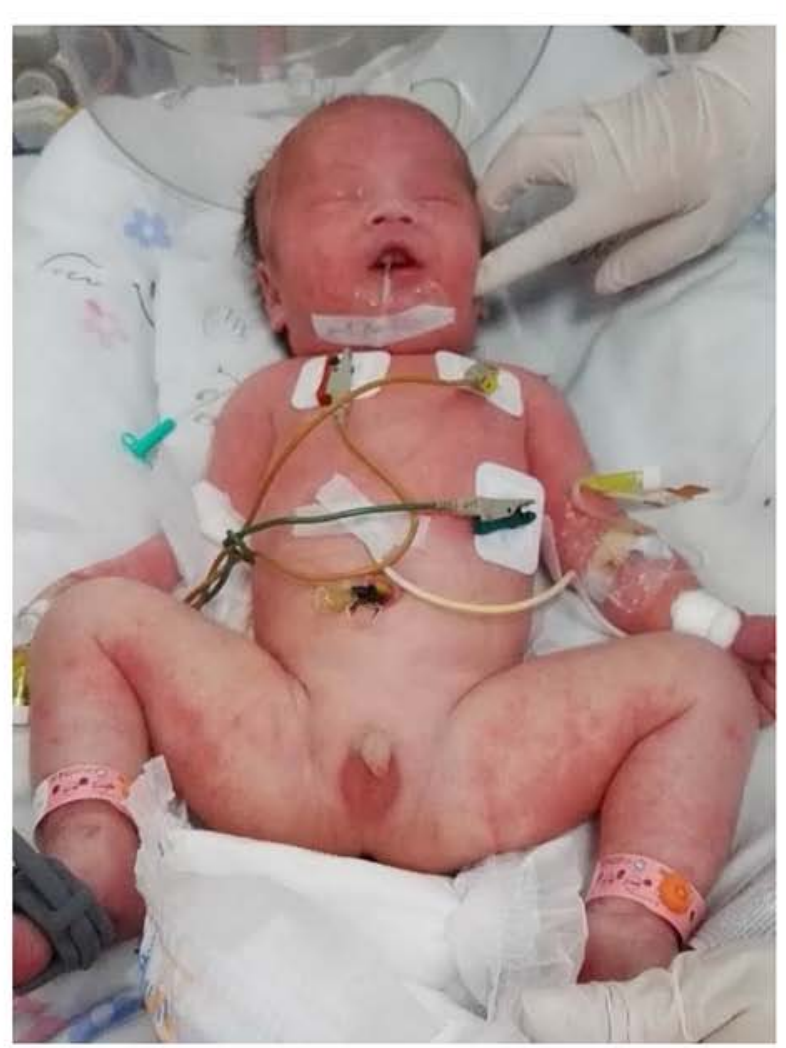

C

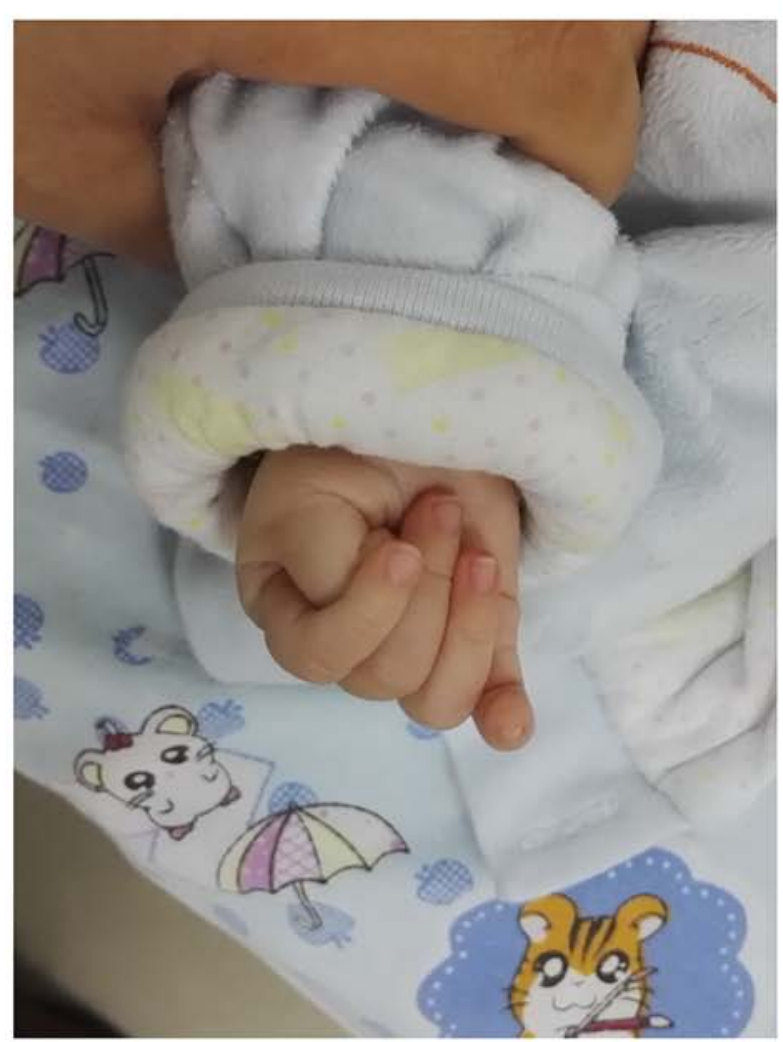

B

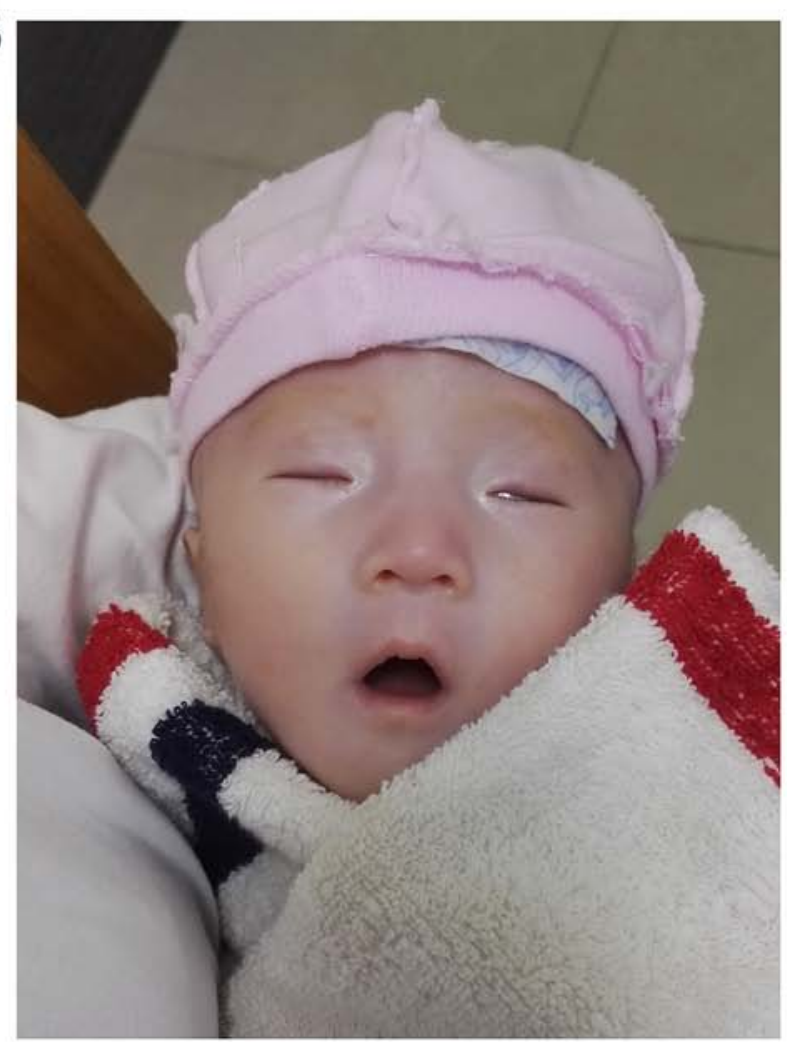

D

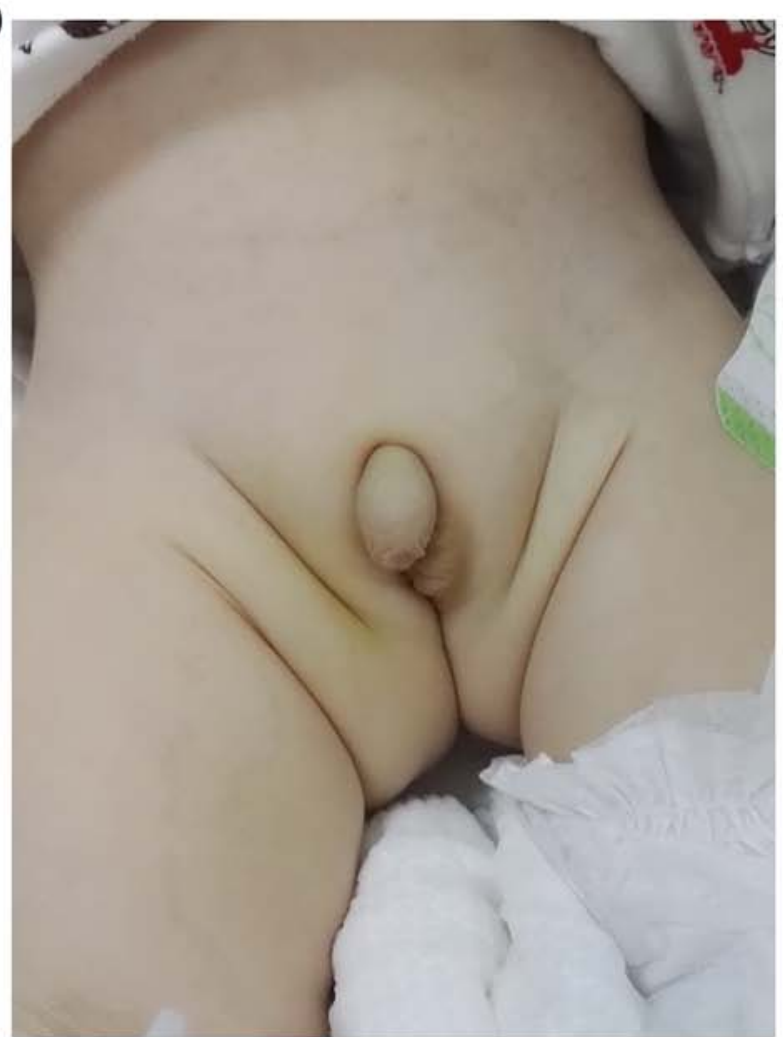

Figure 1. Clinical features of the patient with PWS. (A) Full-length photo of the patient 3 days after birth. (B) Typical facial features of the patient with PWS, including narrow forehead, almond-shaped eyes, thin upper lip, downturned corner of the mouth, fair skin and hair compared with normal infants. Image taken 2 months after birth. (C) Abnormal position of fingers with adducted thumb in the neonatal period, abnormal position of fingers with thumbs adducted over index and middle fingers and persistently clenched hands. Image taken 3 months after birth. (D) Genital hypoplasia with small penis, right cryptorchidism and poorly rugated scrotum. Image taken 3 months after birth. PWS, Prader-Willi syndrome.

version 0.2) (14) was used to transform the exported genotypes into the required format, followed by the detection and classification of the UPD with a UPD tool (http://www. uni-tuebingen.de/uni/thk/de/f-genomik-software.html). 


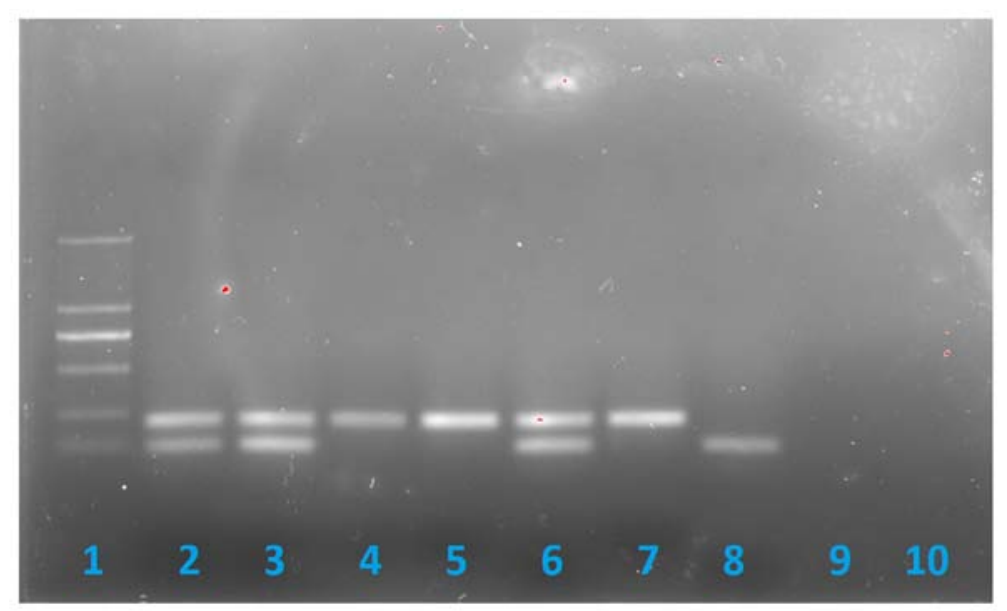

Figure 2. Methylation specific-PCR showing that the patient lacks the paternal $100 \mathrm{bp}$ band. Lane 1, DNA ladder marker; lane 2, father of the patient; lane 3 , mother of the patient; lane 4, patient; lane 5, Prader-Willi syndrome positive control (deletion type); lane 6, negative control; lane 7, PWS positive control (UPD type); lane 8, Angelman syndrome positive control; lane 9, blank control; Lane 10, internal control (non-methylated DNA).

Short tandem repeat (STR) linkage analysis. The aforementioned abnormal findings were subject to further verification, a multiplex-fluorescence-labeled STR assay with DNA from the proband and the parents was performed. Using microsatellite markers from chromosome 15 as the parameters for linkage analysis, including D15S11, D15S646, D15S817, D15S128, D15S1513, GABRB3, D15S822 [typical for PWS/Angelman syndrome (AS) deletion region], D15S659 and FES proto-oncogene, tyrosine kinase (distal region). The experiment was carried out according to the procedure as described in a previous study (15). Data collection and allele sizing were completed using GENEMAPPER software (version 2.2; Applied Biosystems; Thermo Fisher Scientific, Inc.); two STR markers were required as the minimum for identification of the abnormal genotypes.

\section{Results}

Methylation specific PCR. Non-quantitative methylation-specific PCR for the PWS/AS region demonstrated the absence of the paternally derived allele, with only a single band from the maternal allele present (Fig. 2), indicating a total loss of paternal imprinting, typically associated with PWS.

Chromosomal microarray analysis. For single nucleotide polymorphism (SNP)-based CMA, a total of 276,527 markers were compared between the child and parents. The SNP array analysis of the family did not identify any copy number alterations genome-wide. However, trio analysis of SNP loci on chromosome 15 was consistent with uniparental inheritance, and the classification of UPD using the UPDtool revealed a $100 \%$ match in chromosome 15 between the child and the mother, which is indicative of a maternal heterodisomy for the entire chromosome 15 (Fig. 3). No trisomy or monosomy was found using the SNP array.

STR linkage analysis. Family linkage analysis based on STR analysis showed four markers (D15S11,D15S1513,D15S822 and D15S659), mapping to $15 q 11.2 q 21.1$, with only maternal alleles and the absence of a paternal allele in this case. This result indicates maternal heteroUPD of chromosome 15 (Fig. 4).
In view of the clinical manifestation, karyotype, MS-PCR, CMA and STR based linkage analysis, a diagnosis of PWS resulting from complete maternal heteroUPD of chromosome 15 was confirmed for this patient.

\section{Discussion}

As a sporadic genetic disorder with remarkable developmental consequences, PWS is usually triggered by a paternal deletion or maternal UPD of the chromosome region 15q11-q13. The current genetic epidemiology is based on western populations, according to which $15 \mathrm{q} 11.2-\mathrm{q} 13$ paternal deletion is responsible for 70-75\%, maternal UPD for 25-28\%, and ID for $2-5 \%$ of the cases of PWS (16-18). However, studies of Asian populations reveal a smaller proportion of PWS arising from maternal UPD than has previously been recognized $(5,19-21)$. Furthermore, research on Western populations also revealed significant discrepancies in both the genotypes and phenotypes in cases of PWS arising from maternal UPD compared to those with chromosomal region deletions, exhibiting fewer typical facial phenotypes, a shorter course of gavage feeding, later onset of hyperphagia, significantly higher birth weights and a lesser degree of hypotonia (22). These milder clinical manifestations among maternal UPD patients are usually related to missed diagnosis, and thus should arouse vigilance among pediatricians.

Widely accepted mechanisms for the development of maternal UPD 15 are as follows: i) Trisomy rescue, triggered by the loss of one copy of chromosome 15 in a trisomic fetus; ii) gamete complementation, formed in a disomic egg fertilized by a nullisomic sperm; and iii) post-zygotic duplication $(23,24)$. Trisomy rescue, the most commonly accepted mechanism, may give rise to a number of outcomes in somatic cells mitosis, leading to hetero-UPD (rescue), a normal cell (rescue) or trisomy (no or incomplete rescue). Incomplete rescue of trisomy 15 may lead to the development of mosaic mutations and chromosome rearrangements, which are associated with cases of PWS with maternal UPD. In this case, complete heterozygosity of the DNA markers implies non-disjunction in the maternal meiosis II, which is also consistent with 


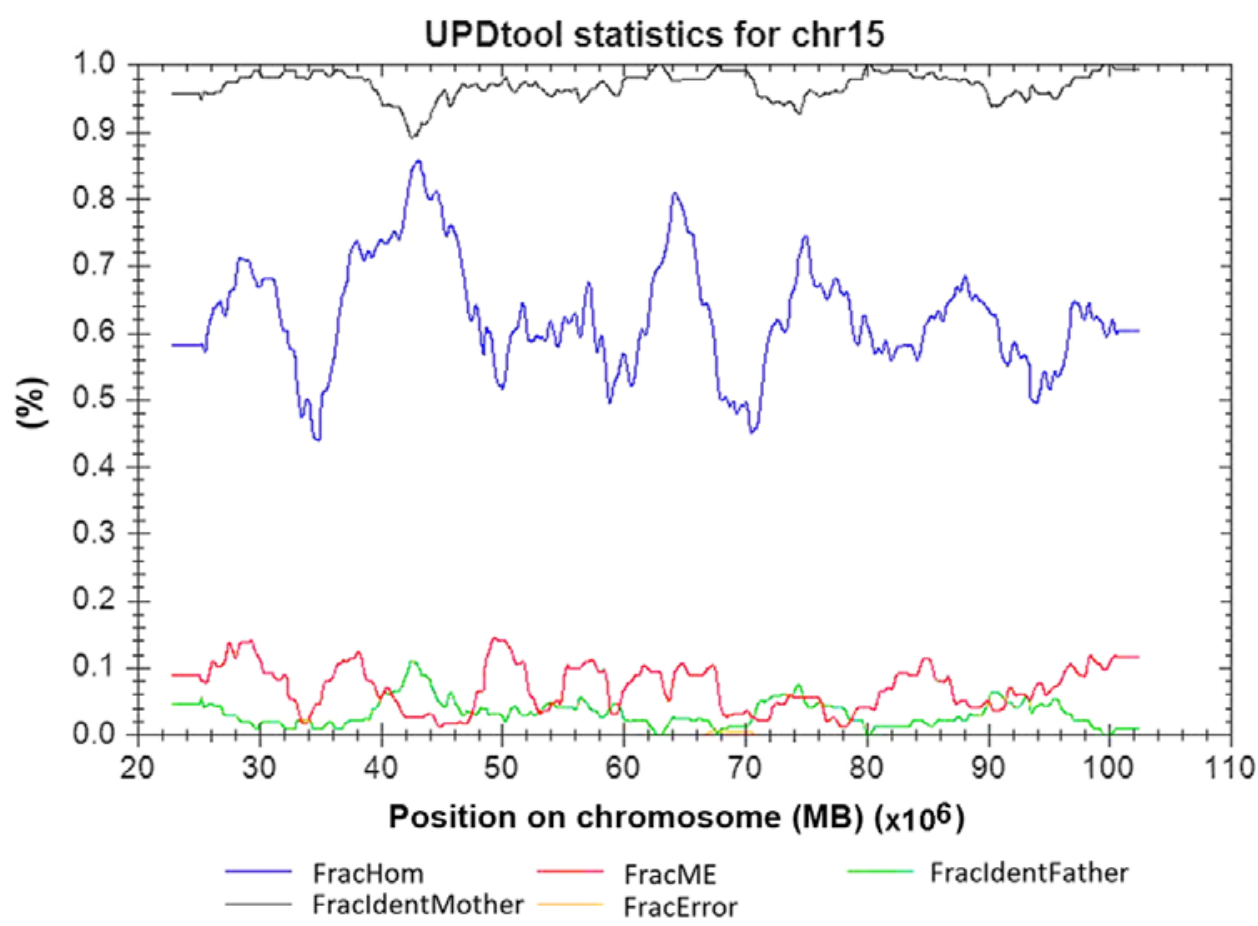

Figure 3. Results of UPDtool analysis for chromosome 15 for the family. The elevated rate of Mendelian errors throughout the chromosomes indicates a UPD. From $\sim 20 \mathrm{Mb}$ to $\sim 100 \mathrm{Mb}$ a high rate of genotypes identical to the mother is typical for a maternal heterodisomy. FracHom, fraction of genotypes in $1 \mathrm{~kb}$-window that is homozygous; FracME, fraction of Mendelian errors in a 1-kb window; FracIdentFather, fraction of genotypes within a 1-kb window where both alleles are identical to the fathers' alleles; FracIdentMother, fraction of genotypes within a $1 \mathrm{~kb}$-window where both alleles are identical to the mothers' alleles; FracError, fraction of errors (Mendelian errors that cannot be explained by UPD) in $1 \mathrm{~kb}$-window. UPD, uniparental disomy.

studies demonstrating that the incidence of chromosome 15 non-disjunction may exponentially increase with maternal age, and that the children of older mothers have an increased rate of maternal UPD for chromosome 15 compared to mothers who are under 35 years of age at delivery $(23,25)$.

Due to the severe complications associated with PWS, it is important to clarify its features so that early diagnoses can be made. At present, PWS is difficult to diagnose prenatally due to a lack of precise and well-characterized fetal phenotypes, which, otherwise, would have provided a basis for further molecular genetic examinations. To date, only a few reports of PWS at the prenatal stage have been published. Most of these reported nonspecific prenatal signs indicative of PWS, and were accidentally identified as trisomy 15 in a routine prenatal examination such as chorionic villus sampling or amniotic fluid culture (26-28), or as a retrospective discovery in postnatal diagnosis according to fetal ultrasound findings and/or postnatal clinical features (7-9,29-33).

Specific prenatal signs are required to allow further molecular genetic examination for PWS. Studies have identified a number of clinical features of the fetus as indicators for PWS, including the abnormal position of feet and toes (9), polyhydramnios $(9,31)$, cerebral anomalies (33), decreased fetal activity $(9,34)$ and hypoplasia of external genitalia $(33,35)$, with the hope that these features can facilitate an early prenatal diagnosis of PWS. However, none of these are representative. In 2008, Bigi et al (9) issued a report in which a possible fetal phenotype was delineated for the first time, including abnormal extremity positions accompanied by reduced fetal movement and polyhydramnios, which are also regarded as informative in the diagnosis of PWS.
Further studies followed these, but there remains an absence of detailed prenatal characteristics of fetuses with PWS. Therefore, prenatally-diagnosed cases of PWS described in the literature have been reviewed in the present report and the major characteristics detailed in Table SI $(9,26,28-41)$. A previous report on a total of 28 prenatal cases with PWS suggests that the phenotypes of the fetuses are variable despite the similarities shared by most of the cases. Among the phenotypic features commonly exhibited in prenatal individuals harboring PWS, the top 10 are breech position $(6 / 7$, $85.7 \%)$, polyhydramnios $(13 / 26,50 \%)$, intrauterine growth restriction (IUGR) $(10 / 26,38.5 \%)$, decreased fetal movement $(8 / 26,30.8 \%)$, facial dysmorphism $(5 / 17,29.4 \%)$, extended legs/feet with flexed toes $(5 / 17,29.4 \%)$, low abdominal circumference $(4 / 16,25 \%)$, low femoral length $(4 / 16,25 \%)$, clenched hands $(3 / 16,18.8 \%)$, hypogonadism $(3 / 16,18.8 \%)$ and low occipital frontal circumference $(2 / 16,12.5 \%)$. Some phenotypic features presented in the present case are consistent with previous reports, including polyhydramnios $(9,30,33)$, breech position (9), clenched hands (33), peculiar position of the extremities $(9,31,33)$ and diminished fetal movement (9). Some of the features, including IUGR and facial dysmorphism, have not been noted or summarized in previous studies to the best of our knowledge. This phenotypic inconsistency was considered to be associated with multiple factors, including the differences in the deletion sizes and affected genes, and even the clinical experience of the obstetricians and ultrasonologists. IUGR and facial dysmorphism, which occur frequently in cases of PWS, should be included as indicators for the prenatal diagnosis of PWS. 


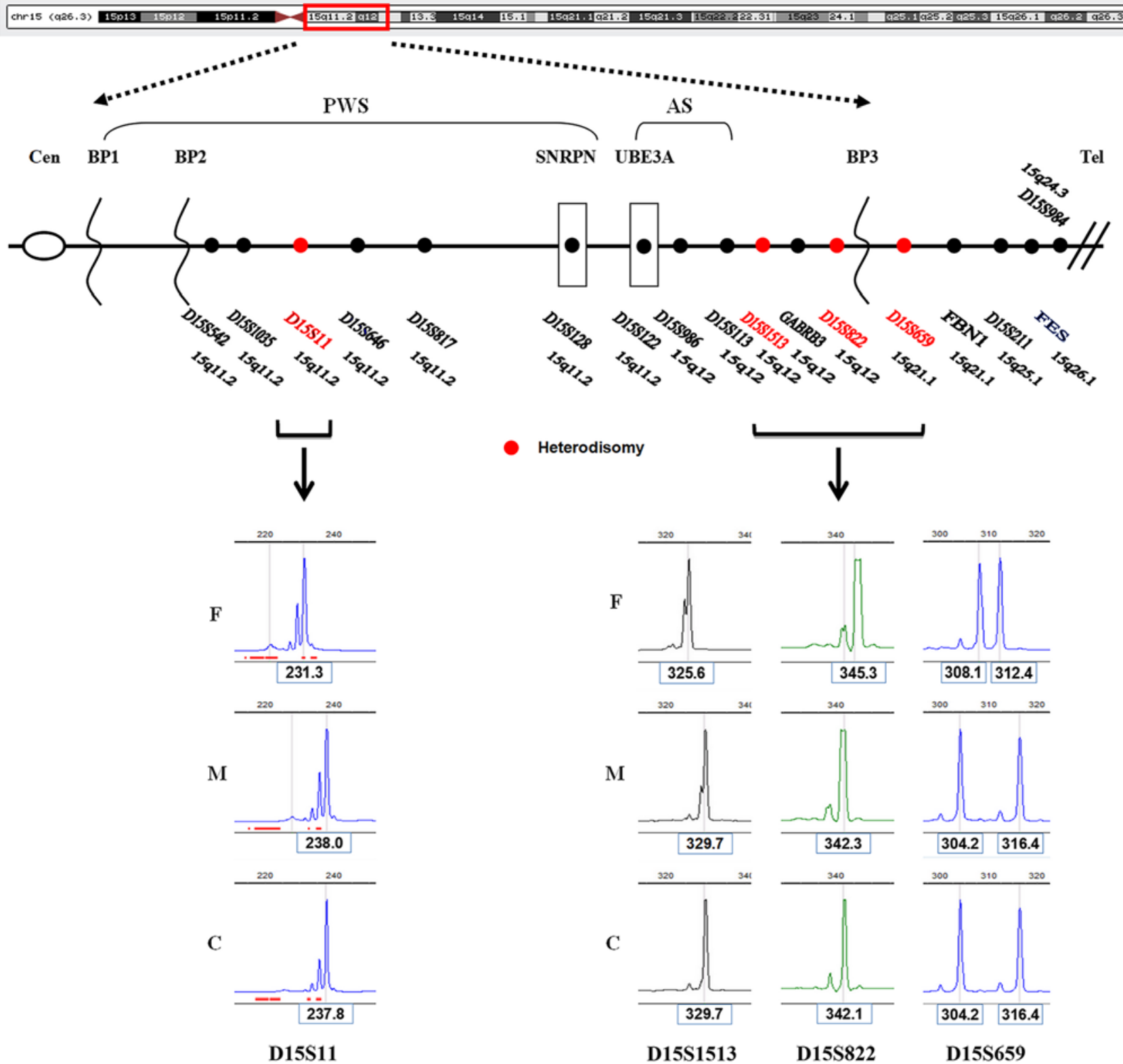

Figure 4. Schematic map of STR based linkage analysis of the patient. Top panel, the PWS/AS critical region is highlighted by a red box. In the middle panel, the locations of nine STR loci alleles (D15S11, D15S646, D15S817, D15S128, D15S1513, GABRB3, D15S822, D15S659, and FES) on 15q are shown as dots, with the different colors denoting the different conditions of the alleles. Seven STR loci alleles (D15S11, D15S646, D15S817, D15S128, D15S1513, GABRB3, and D15S822) are located in the typical PWS/AS critical region of BP1 to BP3, and the remaining two (D15S659 and $F E S$ ) are located in the distal region near the telomere. At the bottom, four electrophoresis maps of STR loci alleles (D15S11, D15S1513, D15S822, and D15S659) reveal that only maternal alleles exist and no paternal allele is passed on to the patient, indicating the maternal heteroUPD of chromosome 15. STR, short tandem repeat; PWS, Prader-Willi syndrome; AS, Angelman syndrome; BP, proximal breakpoint; F, father; M, mother; C, child.

It should be noted that all the indicators have a lack of specificity to allow a confirmed prenatal PWS diagnosis. While a prenatal diagnosis solely relying on ultrasound findings seems implausible, nevertheless, when fetal ultrasound examinations detect these indicators, genetic analysis should be considered to diagnose PWS.

A diagnosis of PWS is established in a proband following a DNA methylation analysis demonstrating abnormal parent-specific imprinting within the Prader-Willi critical region on chromosome 15 in which the region demonstrates maternal-only imprinting. Three molecular mechanisms that give rise to PWS include paternal deletion, maternal UPD15 and ID. Optimizing a diagnostic strategy requires clinicians to fully understand the options available for testing for PWS, including the conditions to use these methods, their technical superiorities and limitations, and the cost of the testing (42). For example, DNA methylation analysis is the only technique able to diagnose PWS caused by the three aforementioned genetic mechanisms and to differentiate PWS from AS in cases of deletion (43). Therefore, DNA methylation analysis is regarded as the primary choice for differentiating PWS from other confounders which cannot be identified using laboratory information, before or after birth. Upon the diagnosis of PWS, further testing is required to explore the underlying mechanism, with the purpose of discriminating the individuals with a high recurrence risk from the larger population with a very low recurrence risk 
(deletions and maternal UPD). The genetic subtypes and testing methods used in the diagnosis of Prader-Willi syndrome are summarized in Table SII. As a universally applied tool for CNV detection, CMA has substituted karyotype analysis and some other specific tests as one of the first choices for patients with developmental/intellectual disorders, autism, congenital abnormalities and other dysmorphic features (44-47). The Affymetrix CytoScan HD array has great value in identifying human chromosomal aberrations due to its broad genomic coverage, and is therefore deemed to be a reliable method capable of specifically detecting 25 - to $50-\mathrm{kb}$ copy number changes across the genome with allelic SNP call corroboration. However, although CMA using the CytoScan HD has proved to be successful in identifying maternal UPD patients, including all iso-UPD cases and most of the iso/hetero-UPD cases, it is likely to fail in the identification of complete hetero-UPD. In the case reported in this present study, the diagnosis was missed in the fetal ultrasound examination, chromosome karyotype analysis and CMA conducted during the pregnancy, and identified retrospectively by postnatal examinations according to fetal ultrasound findings, postnatal clinical features, parent-child trio CMA-SNP array and STR based linkage analysis (48).

As far as the present study is concerned, a failure to diagnosis PWS occurred in prenatal ultrasound and routine prenatal CMA examinations due to the technical limitations of these approaches for detecting complete hetero-UPD. It was the postnatal clinical features that allowed a diagnosis of PWS, which was determined by trio CMA analysis and a multiplex-fluorescence-labeled STR assay developed on the basis of linkage analysis as a rapid and economic detection method for chromosomal region deletion or maternal UPD15. It is now important to determine which approach to the prenatal testing of PWS is sufficient enough to make confirmed diagnoses. Certain clinical features of the fetus, including anomalous extremity positions and subdued fetal movement, combined with polyhydramnios, could be considered indicators of the need for further testing to allow the early prenatal diagnosis of PWS owing to the high potential risk of PWS and the technical limitation of CMA. If clinical signs strongly suggest PWS (abnormal ultrasonic results and advanced maternal age), methylation and/or UPD analysis is highly recommendable.

\section{Acknowledgements}

Not applicable.

\section{Funding}

The present study was financially supported by National Natural Science Foundation of China for Youth (grant no. 31501207) and Research Project of Traditional Chinese Medicine Bureau of Guangdong Province (grant no. 20171030). We also would like to appreciate the support from '111 program' of Ministry of Education P.R.C and State Administration of Foreign Experts Affairs P.R.C.

\section{Availability of data and materials}

The datasets used and/or analyzed during the current study are available from the corresponding author on reasonable request.

\section{Authors' contributions}

YD and SL evaluated the patients clinically and wrote the manuscript. JuL and JiL performed the prenatal ultrasonic examinations and amniocentesis. QC and JYL worked on cell culture of amniotic fluid and karyotype analysis. CL and HL undertook molecular cytogenetic testing and bioinformatics analysis. HQ and RL acted as the lead clinician in the study, counseled the parents, and guided the writing of the manuscript.

\section{Ethics approval and consent to participate}

The present work was approved by The Ethics Committee of The First Affiliated Hospital of Chongqing Medical University and The Ethics Committee of Guangdong Women and Children Hospital. Written consent was given by the parents for full photographs, clinical and laboratory studies. Blood samples used as positive and negative controls were collected from patients with PWS and healthy subjects, respectively. All subjects enrolled in the present study signed a written informed consent. All the procedures performed in the present study were in accordance with the Declaration of Helsinki.

\section{Patient consent for publication}

Written informed consent was obtained from the parents of all patients for the publication of all associated data and images.

\section{Competing interests}

The authors declare that they have no competing interests.

\section{References}

1. Prader A, Labhart A and Willi H: Ein Syndrom von Adipositas, Kleinwuchs, Kryptorchismus und Oligophrenie nach myotonieartigem Zustand im Neugeborenalter. Schweir Med Wschr 86: 1260-1261, 1956

2. Oiglane-Shlik E, Zordania R, Varendi H, Antson A, Mägi ML, Tasa G, Bartsch O, Talvik $\mathrm{T}$ and Ounap K: The neonatal phenotype of Prader-Willi syndrome. Am J Med Genet A 140: 1241-1244, 2006.

3. Hirsch HJ,Eldar-Geva T, Bennaroch F, Pollak Y and Gross-Tsur V: Sexual dichotomy of gonadal function in Prader-Willi syndrome from early infancy through the fourth decade. Hum Reprod 30: 2587-2596, 2015

4. Hartin SN, Hossain WA, Weisensel N and Butler MG: Three siblings with Prader-Willi syndrome caused by imprinting center microdeletions and review. Am J Med Genet A 176: 886-895, 2018.

5. Ramsden SC, Clayton-Smith J, Birch R and Buiting K: Practice guidelines for the molecular analysis of Prader-Willi and Angelman syndromes. BMC Med Genet 11: 70, 2010.

6. Horsthemke B and Wagstaff J: Mechanisms of imprinting of the Prader-Willi/Angelman region. Am J Med Genet A 146A: 2041-2052, 2008.

7. Geysenbergh B, De Catte L and Vogels A: Can fetal ultrasound result in prenatal diagnosis of Prader-Willi syndrome? Genet Couns 22: 207-216, 2011.

8. Whittington JE, Butler JV and Holland AJ: Pre-, peri- and postnatal complications in Prader-Willi syndrome in a UK sample. Early Hum Dev 84: 331-336, 2008.

9. Bigi N, Faure JM, Coubes C, Puechberty J, Lefort G, Sarda P and Blanchet P: Prader-Willi syndrome: Is there a recognizable fetal phenotype? Prenat Diagn 28: 796-799, 2008.

10. Apgar V: A proposal for a new method of evaluation of the newborn infant. Curr Res Anesth Analg 32: 260-267, 1953.

11. Butterfield $J$ and Covey MJ: Practical epigram of the Apgar score. JAMA 181: 353, 1962. 
12. Hussain Askree S, Hjelm LN, Ali Pervaiz M, Adam M, Bean LJ, Hedge $\mathrm{M}$ and Coffee B: Allelic dropout can cause false-positive results for Prader-Willi and Angelman syndrome testing. J Mol Diagn 13: 108-112, 2011.

13. Kubota T, Das S, Christian SL, Baylin SB, Herman JG and Ledbetter DH: Methylation-specific PCR simplifies imprinting analysis. Nat Genet 16: 16-17, 1997.

14. Schroeder C, Sturm M, Dufke A, Mau-Holzmann U, Eggermann T, Poths S, Riess O and Bonin M: UPDtool: A tool for detection of iso- and heterodisomy in parent-child trios using SNP microarrays. Bioinformatics 29: 1562-1564, 2013.

15. Zhang K, Liu S, Feng B, Yang Y, Zhang H, Dong R, Liu Y and Gai Z: Clinical application of an innovative multiplex-fluorescent-labeled STRs assay for Prader-Willi syndrome and angelman syndrome. PLoS One 11: e0147824, 2016.

16. Shaffer LG, Ledbetter DH and Lupski JR: Molecular cytogenetics of contiguous gene syndromes: Mechanisms and consequences of gene dosage imbalance. In: The metabolic and molecular bases of inherited disease (Scriver CR, Beaudet AL, Sly WS, Valle D, eds). New York, McGraw-Hill, pp1291-1324, 2001.

17. Jin DK: Systematic review of the clinical and genetic aspects of Prader-Willi syndrome. Korean J Pediatr 54: 55-63, 2011.

18. Cassidy SB and Driscoll DJ: Prader-Willi syndrome. Eur J Hum Genet 17: 3-13, 2009.

19. Hou JW and Wang TR: Prader-Willi syndrome: Clinical and molecular cytogenetic investigations. J Formos Med Assoc 95: 474-479, 1996.

20. Kim HJ, Cho HJ, Jin DK, Kwon EK, Ki CS, Kim JW and Kim SH: Genetic basis of Prader-Willi syndrome in Korea: Less uniparental disomy than has been recognized? Clin Genet 66: 368-372, 2004

21. Lin HY, Lin SP, Chuang CK, Chen MR, Yen JL, Lee YJ, Huang CY, Tsai LP, Niu DM, Chao MC and Kuo PL: Genotype and phenotype in patients with Prader-Willi syndrome in Taiwan. Acta Paediatr 96: 902-905, 2007.

22. Cassidy SB, Forsythe M, Heeger S, Nicholls RD, Schork N, Benn P and Schwartz S: Comparison of phenotype between patients with Prader-Willi syndrome due to deletion $15 \mathrm{q}$ and uniparental disomy 15. Am J Med Genet 68: 433-440, 1997.

23. Fridman $C$ and Koiffmann CP: Origin of uniparental disomy 15 in patients with Prader-Willi or Angelman syndrome. Am J Med Genet 94: 249-253, 2000

24. Kotzot D: Complex and segmental uniparental disomy (UPD): Review and lessons from rare chromosomal complements. J Med Genet 38: 497-507, 2001

25. Ginsburg C, Fokstuen S and Schinzel A: The contribution of uniparental disomy to congenital development defects in children born to mothers at advanced childbearing age. Am J Med Genet 95: 454-460, 2000.

26. Morichon-Delvallez N, Mussat P, Dumez Y and Vekemans M: Trisomy 15 in chorionic villi and Prader-Willi syndrome at birth Prenat Diagn 13: 307-308, 1993.

27. Christian SL, Smith AC, Macha M, Black SH, Elder FF, Johnson JM, Resta RG, Surti U, Suslak L, Verp MS and Ledbetter DH: Prenatal diagnosis of uniparental disomy 15 following trisomy 15 mosaicism. Prenat Diagn 16: 323-332, 1996.

28. Roberts E, Stevenson K, Cole T, Redford DH and Davison EV: Prospective prenatal diagnosis of Prader-Willi syndrome due to maternal disomy for chromosome 15 following trisomic zygote rescue. Prenat Diagn 17: 780-783, 1997.

29. Hiroi H, Kozuma S, Hayashi N, Unno N, Fujii T, Tsutsumi O, Okai T and Taketani Y: A fetus with Prader-Willi syndrome showing normal diurnal rhythm and abnormal ultradian rhythm on heart rate monitoring. Fetal Diagn Ther 15: 304-307, 2000.

30. Fong BF and De Vries JI: Obstetric aspects of the Prader-Willi syndrome. Ultrasound Obstet Gynecol 21: 389-392, 2003.

31. L'Herminé AC, Aboura A, Brisset S, Cuisset L, Castaigne V, Labrune P, Frydman R and Tachdjian G: Fetal phenotype of Prader-Willi syndrome due to maternal disomy for chromosome 15. Prenat Diagn 23: 938-943, 2003.

32. Haugen G, Rønnestad A and Kroken M: Variations in fetal phenotype in Prader-Willi syndrome. Prenat Diagn 29: 294, 2009.

33. Akiba Y, Ono M, Shirahashi M, Noda S, Nishijima S, Amagata T, Kusano R, Fuke T, Hayashida S, Ikeda T, et al: Polyhydramnios associated with Prader-Willi syndrome. J Obstet Gynaecol 35: $752-753,2015$.
34. Insoft RM, Hurvitz J, Estrella E and Krishnamoorthy KS: Prader-Willi syndrome associated with fetal goiter: A case report. Am J Perinatol 16: 29-31, 1999.

35. Le Bris-Quillevere MJ, Riviere D, Pluchon-Riviere E, Chabaud JJ, Parent P, Volant A and Boog G: Prenatal diagnosis of del(15)(q11q13). Prenat Diagn 10: 405-411, 1990.

36. Bar C, Diene G, Molinas C, Bieth E, Casper C and Tauber M: Early diagnosis and care is achieved but should be improved in infants with Prader-Willi syndrome. Orphanet J Rare Dis 12: 118 , 2017.

37. Liehr T, Brude E, Gillessen-Kaesbach G, König R, Mrasek K, von Eggeling $\mathrm{F}$ and Starke H: Prader-Willi syndrome with a karyotype 47,XY,+min(15)(pter->q11.1:) and maternal UPD 15 -case report plus review of similar cases. Eur J Med Genet 48: 175-181, 2005.

38. Slater HR, Vaux C, Pertile M, Burgess T and Petrovic V: Prenatal diagnosis of Prader-Willi syndrome using PW71 methylation analysis-uniparental disomy and the significance of residual trisomy 15. Prenat Diagn 17: 109-113, 1997.

39. Milunsky JM, Wyandt HE, Huang XL, Kang XZ, Elias ER and Milunsky A: Trisomy 15 mosaicism and uniparental disomy (UPD) in a liveborn infant. Am J Med Genet 61: 269-273, 1996.

40. Cassidy SB, Lai LW, Erickson RP, Magnuson L, Thomas E, Gendron R and Herrmann J: Trisomy 15 with loss of the paternal 15 as a cause of Prader-Willi syndrome due to maternal disomy. Am J Hum Genet 51: 701-708, 1992.

41. Purvis-Smith SG, Saville T, Manass S, Yip MY,Lam-Po-Tang PR, Duffy B, Johnston H, Leigh D and McDonald B: Uniparental disomy 15 resulting from "correction" of an initial trisomy 15 Am J Hum Genet 50: 1348-1350, 1992.

42. Driscoll DJ, Miller JL, Schwartz S, et al: Prader-Willi Syndrome. 1998 Oct 6 (Updated 2017 Dec 14). In: Adam MP, Ardinger HH, Pagon RA, et al, (eds). GeneReviews ${ }^{\circledR}$ [Internet]. Seattle (WA), University of Washington, Seattle, 1993-2018. Available from: https://www.ncbi.nlm.nih.gov/books/NBK1330/.

43. Glenn CC, Driscoll DJ, Yang TP and Nicholls RD: Genomic imprinting: Potential function and mechanisms revealed by the Prader-Willi and Angelman syndromes. Mol Hum Reprod 3: 321-332, 1997.

44. Miller DT, Adam MP, Aradhya S, Biesecker LG, Brothman AR, Carter NP, Church DM, Crolla JA, Eichler EE, et al: Consensus statement: Chromosomal microarray is a first-tier clinical diagnostic test for individuals with developmental disabilities or congenital anomalies. Am J Hum Genet 86: 749-764, 2010.

45. Kearney HM, Thorland EC, Brown KK, Quintero-Rivera F and South ST; Working Group of the American College of Medical Genetics Laboratory Quality Assurance Committee: American College of Medical Genetics standards and guidelines for interpretation and reporting of postnatal constitutional copy number variants. Genet Med 13: 680-685, 2011.

46. Hanemaaijer NM, Sikkema-Raddatz B, van der Vries G, Dijkhuizen T, Hordijk R, van Essen AJ, Veenstra-Knol HE, Kerstjens-Frederikse WS, Herkert JC, Gerkes EH, et al: Practical guidelines for interpreting copy number gains detected by high-resolution array in routine diagnostics. Eur J Hum Genet 20: 161-165, 2012.

47. South ST, Lee C, Lamb AN, Higgins AW and Kearney HM; Working Group for the American College of Medical Genetics and Genomics Laboratory Quality Assurance Committee: ACMG Standards and Guidelines for constitutional cytogenomic microarray analysis, including postnatal and prenatal applications: Revision 2013. Genet Med 15: 901-909, 2013.

48. Tucker T, Schlade-Bartusiak K, Eydoux P, Nelson TN and Brown L: Uniparental disomy: Can SNP array data be used for diagnosis? Genet Med 14: 753-756, 2012.

This work is licensed under a Creative Commons Attribution-NonCommercial-NoDerivatives 4.0 International (CC BY-NC-ND 4.0) License. 